\title{
Prevalence of peri-implant disease on platform switching implants: a cross-sectional pilot study
}

\section{Andrés Duque DUQUE(a) Astrid Giraldo ARISTIZABAL(a) Susana LONDOÑO ${ }^{(a)}$ Lida CASTRO(a) Luis Gonzalo ALVAREZ(b)}

(a) Universidad CES, Faculty of Dentistry, Department of Periodontics, Medellin, Antioquia, Colombia.

(b) Universidad CES, Faculty of Dentistry, Medellin, Antioquia, Colombia.
Declaration of Interests: The authors certify that they have no commercial or associative interest that represents a conflict of interest in connection with the manuscript.

\section{Corresponding Author:}

Andrés Duque Duque

E-mail: aduqued@ces.edu.co

DOI: 10.1590/1807-3107BOR-2016.vol30.0005

Submitted: Apr 15, 2015

Accepted for publication: Jul 28, 2015

Last revision: Sep 03, 2015

\begin{abstract}
The objective of this study was to assess the prevalence of mucositis and peri-implantitis associated with the use of two types of implants-conventional versus platform switching after one year of loading. A longitudinal study of 64 implants in 25 patients was performed. Clinical variables, such as clinical pocket depth and bleeding upon probing, plaque, mobility, gingival recession, clinical attachment loss, and radiographic bone loss, were analyzed. The case definition for peri-implantitis was established as pockets of $\geq 5 \mathrm{~mm}$ with bleeding and bone loss $\geq 2 \mathrm{~mm}$. One year after implant loading, the prevalence of mucositis and peri-implantitis with conventional implants (CIs) was $81.2 \%$ and $15.6 \%$, respectively. For platform switching implants (PSIs) the prevalence was $90 \%$ and $6.6 \%$, respectively. These differences were not statistically significant $(\mathrm{p}=0.5375)$. However, there was a trend towards a lower prevalence of peri-implantitis with platform switching Implants.
\end{abstract}

Keywords: Dental Implants; Prevalence; Peri-Implantitis.

\section{Introduction}

Currently, the functional and aesthetic restoration of partially or totally edentulous areas with dental implants is widely accepted. Survival rates for dental implants evaluated in several systematic reviews and meta-analyses are $>90 \%$ and depend on the time of evaluation. Success rates are generally lower and are controversial owing to a lack of homogeneity in case definition criteria or lack of reporting. ${ }^{1,2}$

Modifications to conventional implant system (CIs) have been proposed in search of better restorative, aesthetic, and biological results. Platform switching implants system (PSIs) utilize an abutment with a smaller diameter aim to preserve the crestal bone level., ${ }^{3,4}$ Most studies have compared the radiographic bone level between PSIs and CIs. Some studies have proposed that crestal bone stability is a consequence of the internal displacement of the abutment-implant junction, which displaces the inflammatory infiltrate away from the crestal bone and creates a space for the formation of biologic width. $3,4,5,6$

PSIs may preserve the marginal bone by decreasing the forces around the implant concentration of forces inward, resulting in the preservation of crestal bone level. ${ }^{7}$ Some studies suggest that there are no significant differences in crestal bone level among different platform designs. ${ }^{6,7,8,9}$ 
The success of dental implants is evidenced by their permanence over the active load period under ideal conditions, limiting crestal bone loss, pocket formation, marginal bleeding, and recession..$^{78}$ The Sixth European Workshop's consensus on periodontics reported a prevalence of mucositis in $50 \%$ of implants $(80 \%$ of patients) and a prevalence of peri-implantitis in $12-40 \%$ of implants (28-56\% of patients). ${ }^{10}$ The controversy regarding the prevalence is due to many factors, such as the case definition used, the characteristics of the population evaluated, and the follow-up period. Numerous studies have shown the impact of PSIs on the preservation of crestal bone level, ${ }^{11,12,13,14}$ but few studies have focused on the impact of the implant-abutment connection and the onset of mucositis and peri-implantitis.

The aim of this study was to test the hypothesis that PSIs are positively associated with better clinical parameters. We evaluated the prevalence of mucositis and peri-implantitis in two different types of implants (PSI vs. CI) after one year of loading. Additionally, differences in plaque index, clinical probing depth (PD), clinical attachment loss (CAL), gingival recession (REC), bleeding, suppuration, and crestal bone level were evaluated.

\section{Methodology}

A cross-sectional study was conducted to assess the prevalence of mucositis and peri-implantitis. Patients treated at Universidad CES (Medellin, Colombia) between 2011 and 2014 were evaluated if they met the inclusion criteria and agreed to participate in the study after signing the informed consent. Inclusion criteria included: periodontally healthy patients with at least one $\mathrm{CI}$ and one PSI placed in the same surgery at least one year ago. Exclusion criteria were: uncontrolled systemic disease, such as diabetes, osteoporosis, smoking, periodontitis or a personal history of radiotherapy. This study was approved by the regional ethics committee under Article 11 of Resolution 8430 of 1993 governing the terms for research on living beings in Colombia and the declaration of Helsinki. The Institutional Ethics Committee approval number was 230 . The sample size of this study was 118 implants in 49 patients who had received both implant designs, 64 implants in 25 patients were followed clinically and radiographically for the pilot study (Figure).

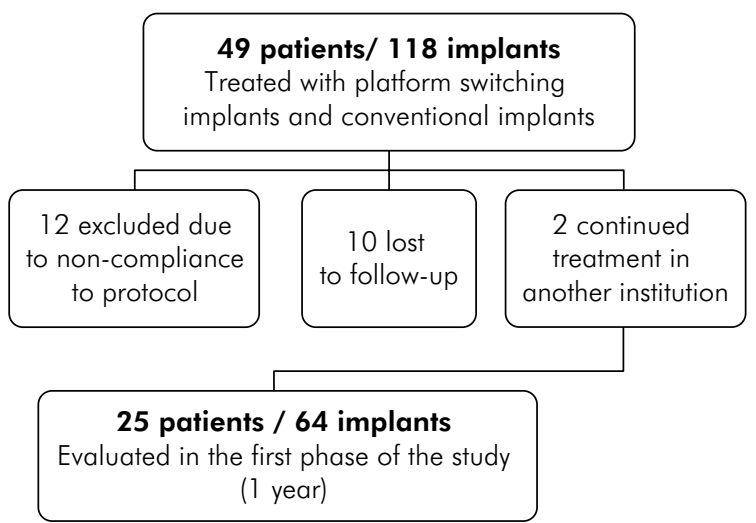

Figure. Flowchart.

Two different implant-abutment connections were studied. A PSIs was used in the test group (The Certain PREVAIL implant, BIOMET 3i, Palm Beach Gardens, USA), and CIs was used for the control group (OSSEOTITE Certain implant, BIOMET 3i, Palm Beach Gardens, USA). The implants were placed randomly by three operators previously trained in the surgical technique and employed in the dental clinic of Universidad CES in Medellin, Colombia.

\section{Clinical evaluation}

A trained examiner evaluated clinical parameters one year after implant loading. The inter-examiner concordance for quantitative variables showed an interclass correlation coefficient of 0.85 for clinical probing depth. Six sites per implant were evaluated with probe PCPUNC156 from Hu-friedy. The primary outcome variable was clinical probing depth (PD), and the secondary measures were bleeding upon probing, gingival recession (REC), clinical attachment loss (CAL), suppuration, mobility, plaque index, type of restoration (provisional or definitive), type of fixation (screwed or cemented), and prosthesis design (unitary or splinted implants).

\section{Radiographic evaluation}

Periapical images of each of the implants at the baseline and one year after implant loading were obtained. A customized parallel technique for the analysis of the crestal bone level was used. The effective radiation dose was $5 \mathrm{mSv}$ per radiograph. The equipment used for scanning images was 
Vistascann perio from the producer Dental Durr of Germany, and the software for measuring and analysis was DBSWIN. A radiology assistant previously standardized for evaluation of radiographic variables used a plastic Rinn block with an acrylic bite to record the incisal/occlusal position of the provisional or definitive implant crown. This was done to ensure a good radiographic orientation, and the image magnification was constant. The radiographic method used periapical images obtained on photostimulable phosphor plates, which allow a resolution of up to $40 \mathrm{LP} / \mathrm{mm}$. The quality of the final image was evaluated following the radiology center's quality assurance protocol. An unbiased examiner evaluated all radiographs using standard operating procedures to ensure accuracy and precision. The examiner measured the coronal-apical level of the crestal bone and its relation to the proximal surfaces (mesial and distal) in each implant, obtaining the distance from the implant's shoulder to the first bone-implant contact, which was the starting point of the initial radiograph. ${ }^{15,16}$ Radiographs were evaluated with the software DBSWIN to calculate the corresponding lengths based on changes in the crestal bone level. Patients diagnosed with peri-implantitis were referred to a periodontist for appropriate treatment.

\section{Statistical analysis}

Data analysis was carried out with SPSS statistical software version 21.0 (SPSS Inc., Chicago, USA). All quantitative variables (age, probing depth, radiographic bone loss, gingival recession, and clinical attachment loss) were summarized and presented as the mean \pm standard deviation. The qualitative variables (gender, mobility, bleeding upon probing, suppuration, plaque, prosthetic design and fixation of restoration) were summarized and presented as absolute and relative frequencies, with the latter expressed in percentages. Qualitative variables (gender, mobility, bleeding upon probing, suppuration, plaque, prosthetic design and fixation of restoration) are presented as absolute and relative frequencies, with the latter expressed in percentages. Owing to the limitations of the study's sample size, an exploratory analysis was performed to compare the clinical and radiographic variables between the two types of implants using the Student's t-test for independent samples. Owing to the limitations of our sample size, we limited our study to an exploratory analysis that compared the clinical and radiographic variables between the two types of implants using the Student's t-test for independent samples. For all statistical tests, a p-value of $<0.05$ was considered statistically significant. Finally, proportion indicators were used to determine the prevalence of mucositis and peri-implantitis for each type of implant.

\section{Results}

In this study, 64 implants were evaluated as the unit of analysis in 25 patients. Patient ages ranged from 33 to 84 (mean: $54 \pm 12$ years), and there was a predominance of females $(72 \%)$.

Of the 64 implants, 33 (52\%) were in the control group and $31(49 \%)$ were in the test group. No implants showed mobility. There were no significant differences in the type of restoration, prosthetic design, mounting type, length, or diameter of the implants between the two systems (PSI and CI) at the time of evaluation (Table 1).

There were no significant differences between the implant systems in terms of bleeding upon probing, suppuration, plaque, or prevalence of pockets $\geq 4 \mathrm{~mm}$ with bleeding. The prevalence of pockets $\geq 5 \mathrm{~mm}$ was $24.2 \%$ for CIs and $12.9 \%$ for PSIs, and this difference was not statistically significant. Only one implant in the PSIs group had pockets $\geq 6 \mathrm{~mm}$ (Table 2).

The average depth upon clinical probing, clinical attachment loss, and recession were not statistically different between the two systems. The mean interproximal bone loss in the test group was $1.48 \pm 0.81 \mathrm{~mm}$, and in the control group it was $1.97 \pm 0.90 \mathrm{~mm}$, revealing a statistically significant difference between the two systems (Table 3 ).

The prevalence of mucositis was $72.7 \%$ for the control group and $83.8 \%$ for the test group, and the prevalence of peri-implantitis was $24.3 \%$ and $12.9 \%$, respectively, using only a $5 \mathrm{~mm}$ pocket cutoff. The prevalence was similar for both systems. It did not matter if the implants were screwed or cemented. Whether the implants were screwed or cemented did not impact the results for the prevalence of mucositis. The appearance of mucositis was more prevalent in the unitary prosthetic design for both systems, but 
- Prevalence of peri-implant disease on platform switching implants: a cross-sectional pilot study

Table 1. Conventional Implants (CI) and platform switching implants (PSI) characteristics in terms of length, diameter, design and type of prosthetic restoration.

\begin{tabular}{|c|c|c|c|c|}
\hline \multirow{2}{*}{ Clinical Variables } & $\mathrm{Cl}$ & PSI & Total implants & $p$-value \\
\hline & n (\%) & n (\%) & n (\%) & $X^{2}$ Pearson \\
\hline Type of implant & $33(51.6)$ & $31(48.4)$ & $64(100)$ & - \\
\hline Type of Provisional Restoration & $14(53.8)$ & $12(46.2)$ & $26(40.6)$ & 0.762 \\
\hline Type of Definitive Restoration & $19(50)$ & $19(50)$ & $38(59.4)$ & \\
\hline Unitary implants & $30(50)$ & $30(50)$ & $60(93.7)$ & 0.333 \\
\hline Splinted implants & $3(3.3)$ & $1(3.2)$ & $4(6.3)$ & \\
\hline Cemented Implants & $16(53.3)$ & $14(46.7)$ & $30(46.9)$ & 0.790 \\
\hline Screwed Implants & $17(50)$ & $17(50)$ & $34(53.1)$ & \\
\hline Implants with $8.5 \mathrm{~mm}$ length & $5(50)$ & $5(50)$ & $10(15.6)$ & 0.863 \\
\hline Implants with $10 \mathrm{~mm}$ length & $10(52.6)$ & $9(47.4)$ & $19(29.7)$ & \\
\hline Implants with $11.5 \mathrm{~mm}$ length & $8(44.4)$ & $10(55.6)$ & $18(28.1)$ & \\
\hline Implants with $13 \mathrm{~mm}$ length & $10(58.8)$ & $7(41.2)$ & $17(26.6)$ & \\
\hline Implants with $4 \mathrm{~mm}$ diameter & $24(100)$ & - & $24(37.5)$ & - \\
\hline Implants with $5 \mathrm{~mm}$ diameter & $9(100)$ & - & $9(14.1)$ & - \\
\hline Implants with $4 / 3 \mathrm{~mm}$ diameter & - & $20(100)$ & $20(31.3)$ & - \\
\hline Implants with $5 / 4 \mathrm{~mm}$ diameter & - & $9(100)$ & $9(14.1)$ & - \\
\hline Implants with $6 / 5 \mathrm{~mm}$ diameter & - & $2(100)$ & $2(3.1)$ & \\
\hline
\end{tabular}

Table 2. Clinical parameters after 1 year of loading of conventional implants (CI) and platform switching implants (PSI).

\begin{tabular}{|c|c|c|c|c|}
\hline & $\mathrm{Cl}$ & PSI & Total surfaces & p-value \\
\hline & $\mathrm{n} / \mathrm{N}(\%)$ & $\mathrm{n} / \mathrm{N}(\%)$ & $\mathrm{n} / \mathrm{N}(\%)$ & $X^{2}$ Pearson \\
\hline \multicolumn{5}{|l|}{ Clinical Variables per area } \\
\hline Bleeding on probing & $152 / 198(76.8 \%)$ & $134 / 186(72 \%)$ & $286 / 384(74.5 \%)$ & 0.289 \\
\hline Suppuration & $9 / 198(4.5 \%)$ & $13 / 186(6.9 \%)$ & $22 / 384(5.7 \%)$ & 0.303 \\
\hline Plaque & $120 / 198(60.6 \%)$ & $99 / 186(53.2 \%)$ & $219 / 384(57.0 \%)$ & 0.144 \\
\hline \multicolumn{5}{|l|}{ Clinical Variables per implant } \\
\hline Prevalence of implants that bled in at least one site & $32 / 33(97 \%)$ & $30 / 31(96.8 \%)$ & $62 / 64(96.9 \%)$ & 0.9641 \\
\hline Prevalence of implants with pockets $\geq 4 \mathrm{~mm}$ with bleeding & $14 / 33(42.4 \%)$ & $14 / 31(45.2 \%)$ & $28 / 64(43.8 \%)$ & 0.973 \\
\hline Prevalence of implants with pockets $\geq 5 \mathrm{~mm}$ with bleeding & $8 / 33(24.2 \%)$ & $4 / 31(12.9 \%)$ & 12/64 (18.8\%) & 0.369 \\
\hline Prevalence of implants with pockets $\geq 6 \mathrm{~mm}$ with bleeding & $0 / 33(0 \%)$ & $1 / 31(3.2 \%)$ & $1 / 64(1.6 \%)$ & 0.620 \\
\hline
\end{tabular}

Table 3. Clinical and Radiographic results after 1 year of loading conventional implants (CI) and platform switching Implants (PSI).

\begin{tabular}{lcccc}
\hline \multirow{2}{*}{ Clinical and Radiographic Variables } & $\mathrm{Cl}$ & $\mathrm{PSI}$ & \multicolumn{1}{c}{ Total surfaces } & $\mathrm{p}$-value \\
\cline { 2 - 5 } & $\overline{\mathrm{X}} \pm \mathrm{DE}$ & $\overline{\mathrm{X}} \pm \mathrm{DE}$ & $\overline{\mathrm{X}} \pm \mathrm{DE}$ & $\mathrm{t}$-student \\
\hline Clinical probing depth & $2.7 \pm 0.9$ & $2.6 \pm 1.2$ & $2.7 \pm 1.1$ & 0.296 \\
REC & $0.3 \pm 0,7$ & $0.24 \pm 0,6$ & $0.3 \pm 0,7$ & 0.509 \\
NIC & $-2.5 \pm 1,0$ & $-2.4 \pm 1,2$ & $-2.4 \pm 1,1$ & 0.558 \\
Bone loss (mm) & $1.97 \pm 0,90$ & $1.48 \pm 0,81$ & $-1.73 \pm 0,88$ & 0.030 \\
\hline
\end{tabular}

the difference was not statistically significant. The prevalence of mucositis tended to be greater for the unitary prosthetic design for both systems, but the difference was not statistically significant (Table 4).

\section{Clinical and radiographic variables in the prevalence of mucositis and peri-implantitis}

We carried out clinical and clinical-radiographic analyses to determine the prevalence of mucositis 
and peri-implantitis. For the clinical-radiographic analysis of peri-implantitis, the case definition used was that of Persson et al. ${ }^{17}$

The radiographic analysis, unlike the clinical analysis, could only be performed on 62 implants given that one patient did not attend the radiology center for the final evaluation. There was no significant difference in the prevalence of peri-implantitis or mucositis between the two systems, but a trend was noted towards a lower prevalence of peri-implantitis in the PSIs group (Table 5).

\section{Discussion}

The Sixth European Workshop suggested two definitions for peri-implant diseases that should be adopted in future research: peri-implant mucositis, based on the clinical parameter of bleeding upon probing without loss of bone support, and peri-implantitis, defined as detectable bleeding upon probing and bone loss after one year of loading. ${ }^{10}$ Currently there is controversy over the case definition of peri-implantitis. Different definitions have been reported in the literature. Leonhardt et al..$^{18}$ defined it as bone loss $\geq 3$ threads, the presence of a pocket, and suppuration. Botero et al. ${ }^{19}$ defined it as pockets $\geq 4 \mathrm{~mm}$ with bleeding upon probing. ${ }^{18}$ Schwarz et al. ${ }^{20}$ defined it as bone loss $>3 \mathrm{~mm}$, pockets $>6 \mathrm{~mm}$, and bleeding upon probing. Persson et al..$^{17}$ defined it as bone loss $\geq 2 \mathrm{~mm}$, pockets $\geq 5 \mathrm{~mm}$, and bleeding upon probing. Finally, Persson et al. ${ }^{21}$ defined it as bone loss $>2.5 \mathrm{~mm}$, pockets $\geq 4 \mathrm{~mm}$, and bleeding or suppuration upon probing. The prevalence of peri-implantitis is difficult to determine because of different case definitions and follow-up periods. For example, one study reported a prevalence of $6.61 \%$ over a period of 9-14 years, ${ }^{22}$ another found a prevalence of $23 \%$ during a 10 - year period, ${ }^{23}$ and a third study reported a prevalence of $36.6 \%$ with an average follow-up period of 8.4 years. ${ }^{24}$

Different implant systems have been developed to preserve the crestal bone level, which improves the cosmetic results and reduces biological complications including peri-implant bone loss during the first year of function. ${ }^{5}$ This pilot study is the first to evaluate the differences in clinical parameters between PSIs and CIs. Although some studies have claimed that the PSI system reduces bone loss, ${ }^{3,4,5}$ there are no reports comparing the prevalence of peri-implant diseases

Table 4. Prevalence of mucositis and peri-implantitis using the clinical criteria of pockets $\geq 5 \mathrm{~mm}$ according to implant system (CI vs. PSI), fixation and prosthetic design.

\begin{tabular}{|c|c|c|c|c|c|c|c|c|}
\hline & & \multicolumn{3}{|c|}{$\mathrm{Cl}$} & \multicolumn{3}{|c|}{ PSI } & \multirow{3}{*}{$\mathrm{p}$-value } \\
\hline & & Healthy & Mucositis & Peri-implantitis & Healthy & Mucositis & Peri-implantitis & \\
\hline & & $n(\%)$ & n (\%) & n (\%) & n (\%) & $\mathrm{n}(\%)$ & $\mathrm{n}(\%)$ & \\
\hline \multirow[t]{3}{*}{ Fixed } & Cemented & $0(0)$ & $12(75)$ & $4(25)$ & $1(7.1)$ & $12(85.7)$ & $1(7.1)$ & 0.262 \\
\hline & Screwed & $1(5.9)$ & $12(70.6)$ & $4(23.5)$ & $0(0)$ & $14(82.4)$ & $3(17.6)$ & 0.523 \\
\hline & $\mathrm{p}$-value & 0,921 & & & & & & \\
\hline \multirow{3}{*}{$\begin{array}{l}\text { Prosthetic } \\
\text { Design }\end{array}$} & Unitary & 1 (3.3) & 22 (73.3) & 7 (23.3) & 1 (3.3) & 25 (83.3) & 4 (13.3) & 0.604 \\
\hline & Splinted & $0(0)$ & $2(66.7)$ & 1 (33.3) & $0(0)$ & $1(100)$ & $0(0)$ & 0.505 \\
\hline & $\mathrm{p}$-value & & & & 0.892 & & & \\
\hline
\end{tabular}

Table 5. Prevalence of mucositis and peri-implantitis according to clinical and radiographic parameters with a $5 \mathrm{~mm}$ cutoff and bone loss $\geq 2 \mathrm{~mm}$.

\begin{tabular}{lrrr}
\hline Diagnosis & \multicolumn{1}{c}{$\mathrm{Cl}$} & PSI & Total Implants \\
\cline { 2 - 4 } & $\mathrm{n} / \mathrm{N}(\%)$ & $\mathrm{n} / \mathrm{N}(\%)$ & $\mathrm{n} / \mathrm{N}(\%)$ \\
\hline Healthy (no bleeding with any pocket or sulcus without bone loss) & $1 / 32(3.0 \%)$ & $1 / 30(3.2 \%)$ & $2 / 62(3.2 \%)$ \\
Mucositis (sulcus with bleeding on probing with radiographic bone loss $<2 \mathrm{~mm})$ & $26 / 32(81.25 \%)$ & $27 / 30(90 \%)$ & $53 / 62(85.5 \%)$ \\
Peri-implantitis (sulcus $\geq 5 \mathrm{~mm}$ with bleeding on probing and radiographic bone loss $\geq 2 \mathrm{~mm})$ & $5 / 32(15.6 \%)$ & $2 / 30(6.6 \%)$ & $7 / 62(11.3 \%)$ \\
\hline p-value: 0.5375. & &
\end{tabular}


(mucositis and peri-implantitis) between PSIs and CIs. Our present study used the definition of Persson in 2006 and found a prevalence of peri-implantitis of $15.6 \%$ with CIs and $6.6 \%$ with PSIs. Although the difference was not statistically significant, a trend towards a lower prevalence of peri-implantitis in the test group was observed. Of 118 implants in 49 patients treated with both implant designs, 64 implants in 25 patients were monitored clinically and radiographically. One limitation of this pilot study was the high rate of withdrawals and dropouts. In total, 12 patients did not comply with the protocol and were excluded from the analysis (withdrawals). The main reason was the lack of compliance with the implant loading protocol (6 months) established in the study. An additional 12 patients left the study (dropouts) during the monitoring stage. These findings should be evaluated in further studies with larger samples and longer follow-up periods. The prevalence of peri-implant mucositis was 81.3\% for CIs and $90.0 \%$ for the PSI system.

In the present study, no statistically significant differences were found between the two implant systems with regard to clinical variables, such as bleeding upon probing, depth upon clinical probing, suppuration, plaque index, and mobility. These results are consistent with those of a controlled randomized clinical trial in which no differences in clinical parameters between CIs and PSIs were found. ${ }^{25} \mathrm{~A}$ histological study concluded that both systems had the same histological and soft-tissue characteristics despite changes in bone levels. ${ }^{26}$

In this preliminary report, statistically significant differences in mean radiographic bone loss between the two groups of implants were observed. Several clinical and histological studies have reported that the PSI system results in less crestal bone resorption. ${ }^{6,25}$ A controlled clinical trial reported 30\% less crestal bone loss in implants that had abutments with smaller diameters. After a year of loading, average bone loss was $0.94 \mathrm{~mm}$ for the CI system and $0.66 \mathrm{~mm}$ for the PSI system. ${ }^{27}$ Studies in animals and humans found no difference in crestal bone remodeling in immediate implant abutments with different configurations. ${ }^{15,28}$
Failure to comply with follow-up visits may explain the high percentage of plaque in this study (57\%), in which $96.9 \%$ of the implants had at least one bleeding site. The presence of plaque and bleeding upon probing are associated with an increased risk of peri-implant diseases. ${ }^{29,30}$ Experimental studies on peri-implantitis have shown that increased probing depth (PD) is related to bone loss and clinical attachment loss (CAL). ${ }^{29,30}$

The prevalence of peri-implantitis in this study was $6.6 \%$ with PSIs and $15.6 \%$ with CIs. Although no differences in the prevalence of peri-implant diseases were found, there was one patient who had $\mathrm{a} \geq 6 \mathrm{~mm}$ pocket, in whom radiographically excess cement was found. The retention of cement in the peri-implant sulcus and the restorative margin can sometimes explain the occurrence of these diseases.

In this pilot study, systemically healthy patients, with no history of periodontitis or smoking, were evaluated. The patients had lost their teeth for reasons not associated with periodontitis, such as caries, fractures, or endodontic complications. An increased risk of peri-implantitis occurs in smokers compared with nonsmokers (reported odds ratios from 3.6 to 4.6). The combination of a history of treated periodontitis and smoking increases the risk of implant failure and peri-implant bone loss. ${ }^{31}$ Further studies in different populations with longer follow-up periods should be performed, and the behavior of tissues with different implant designs and other risk factors should be evaluated.

\section{Conclusions}

The prevalence of peri-implant mucositis was $81.2 \%$ for CIs and $90 \%$ for the PSIs. There was a trend towards a lower prevalence of peri-implantitis (not statistically significant) with PSI (6.6\%) compared with CI (15.6\%).

The mean interproximal bone loss in the test group was $1.48 \pm 0.81 \mathrm{~mm}$, whereas in the control group it was $1.97 \pm 0.90 \mathrm{~mm}$, revealing a statistically significant difference between the two systems. There were no statistical differences in clinical parameters. 


\section{References}

1. Den Hartog L, Slater JJRH, Vissink A, Meijer HJA, Raghoebar GM. Treatment outcome of immediate, early and conventional single-tooth implants in the aesthetic zone: a systematic review to survival, bone level, soft-tissue, aesthetics and patient satisfaction. J Clin Periodontol. 2008 Dec;35(12):1073-86.

2. Slagter KW, Den Hartog L, Bakker NA, Vissink A, Meijer HJA, Raghoebar GM. Immediate placement of dental implants in the esthetic zone: a systematic review and pooled analysis. J Periodontol. 2014 Jul;85(7):e241-250.

3. Gardner DM. Platform switching as a means to achieving implant esthetics. N Y State Dent J. 2005 Apr;71(3):34-7.

4. Baumgarten H, Cocchetto R, Testori T, Meltzer A, Porter S. A new implant design for crestal bone preservation: initial observations and case report. Pract Proced Aesthetic Dent. 2005 Nov-Dec;17(10):735-40.

5. Lazzara RJ, Porter SS. Platform switching: a new concept in implant dentistry for controlling postrestorative crestal bone levels. Int J Periodontics Restorative Dent. 2006 Feb;26(1):9-17.

6. Farronato D, Santoro G, Canullo L, Botticelli D, Maiorana C, Lang NP. Establishment of the epithelial attachment and connective tissue adaptation to implants installed under the concept of "platform switching": a histologic study in minipigs. Clin Oral Implants Res. 2012 Jan;23(1):90-4.

7. Maeda Y, Miura J, Taki I, Sogo M. Biomechanical analysis on platform switching: is there any biomechanical rationale? Clin Oral Implants Res. 2007 Oct;18(5):581-4.

8. Calvo-Guirado JL, Gómez-Moreno G, López-Marí L, Guardia J, Negri B, Martínez-González JM. Crestal bone loss evaluation in osseotite expanded platform implants: a 5-year study. Clin Oral Implants Res. 2011 Dec;22(12):1409-14.

9. Baffone GM, Botticelli D, Pantani F, Cardoso LC, Schweikert MT, Lang NP. Influence of various implant platform configurations on peri-implant tissue dimensions: an experimental study in dog. Clin Oral Implants Res. 2011 Apr 22(4):438-44.

10. Lindhe J, Meyle J. Peri-implant diseases: consensus report of the sixth european workshop on periodontology. J Clin Periodontol. 2008 Sep;35(8 Suppl):282-5.

11. Bressan E, Lang NP, Corazza B, Ricci S, Almagro Urrutia Z, Botticelli D. The platform switching concept revisited. An experimental study in dogs. Clin Oral Implants Res. 2014;Oct;25(10):1200-6.

12. Wagenberg B, Froum SJ. Prospective study of 94 platform-switched implants observed from 1992 to 2006. Int J Periodontics Restorative Dent. 2010 Feb;30(1):9-17.

13. Canullo L, Fedele GR, Iannello G, Jepsen S. Platform switching and marginal bone-level alterations: the results of a randomized-controlled trial. Clin Oral Implants Res. 2010 Jan;21(1):115-21.

14. Atieh MA, Ibrahim HM, Atieh AH. Platform switching for marginal bone preservation around dental implants: a systematic review and meta-analysis. J Periodontol. 2010 Oct;81(10):1350-66.
15. Crespi R, Capparè $\mathrm{P}$, Gherlone E. Radiographic evaluation of marginal bone levels around platform-switched and non-platform-switched implants used in an immediate loading protocol. Int J Oral Maxillofac Implants. 2009Sep-Oct;24(5):920-6.

16. Cappiello M, Luongo R, Di Iorio D, Bugea C, Cocchetto R, Celletti R. Evaluation of peri-implant bone loss around platform-switched implants. Int J Periodontics Restorative Dent. 2008 Aug;28(4):347-55.

17. Persson GR, Salvi GE, Heitz-Mayfield LJA, Lang NP. Antimicrobial therapy using a local drug delivery system (Arestin) in the treatment of perimplantitis. I: Microbiological outcomes. Clin Oral Implants Res. 2006 Aug;17(4):386-93.

18. Leonhardt A, Renvert S, Dahlén G. Microbial findings at failing implants. Clin Oral Implants Res. 1999 Oct;10(5):339-45.

19. Botero JE, González AM, Mercado RA, Olave G, Contreras A. Subgingival microbiota in peri-implant mucosa lesions and adjacent teeth in partially edentulous patients. J Periodontol. 2005 Sep;76(9):1490-5.

20. Schwarz F, Sculean A, Rothamel D, Schwenzer K, Georg T, Becker J. Clinical evaluation of an Er:YAG laser for nonsurgical treatment of perimplantitis: a pilot study. Clin Oral Implants Res. 2005 Feb;16(1):44-52.

21. Persson GR, Samuelsson E, Lindahl C, Renvert S. Mechanical non-surgical treatment of perimplantitis: a single-blinded randomized longitudinal clinical study. II. Microbiological results. J Clin Periodontol. 2010 Jun;37(6):563-73.

22. Gerber JA, Tan WC, Balmer TE, Salvi GE, Lang NP. Bleeding on probing and pocket probing depth in relation to probing pressure and mucosal health around oral implants. Clin Oral Implants Res. 2009 Jan;20(1):75-8.

23. Albrektsson T, Zarb G, Worthington P, Eriksson AR. The long-term efficacy of currently used dental implants: a review and proposed criteria of success. Int J Oral Maxillofac Implants. 1986 Summer;1(1):11-25.

24. Heitz-Mayfield LJA, Lang NP. Comparative biology of chronic and aggressive periodontitis vs. perimplantitis. Periodontol 2000. 2010 Jun;53:167-81.

25. Telleman G, Raghoebar GM, Vissink A, Meijer HJA. Impact of platform switching on inter-proximal bone levels around short implants in the posterior region; 1-year results from a randomized clinical trial. J Clin Periodontol. 2012 Jul;39(7):688-97.

26. Canullo L, Pellegrini G, Allievi C, Trombelli L, Annibali S, Dellavia C. Soft tissues around long-term platform switching implant restorations: a histological human evaluation. Preliminary results. J Clin Periodontol. 2011 Jan;38(1):86-94.

27. Vandeweghe S, De Bruyn H. A within-implant comparison to evaluate the concept of platform switching: a randomised controlled trial. Eur J Oral Implantol. 2012 Autumn;5(3):253-62.

28. Becker J, Ferrari D, Mihatovic I, Sahm N, Schaer A, Schwarz F. Stability of crestal bone level at platform-switched non-submerged titanium implants: a histomorphometrical study in dogs. J Clin Periodontol. 2009 Jun;36(6):532-9. 
- Prevalence of peri-implant disease on platform switching implants: a cross-sectional pilot study

29. Lang NP, Brägger U, Walther D, Beamer B, Kornman KS. Ligature-induced peri-implant infection in cynomolgus monkeys. I. Clinical and radiographic findings. Clin Oral Implants Res. 1993 Mar;4(1):2-11.

30. Schou S, Holmstrup P, Stoltze K, Hjørting-Hansen E, Kornman KS. Ligature-induced marginal inflammation around osseointegrated implants and ankylosed teeth. Clin Oral Implants Res. 1993 Mar;4(1):12-22.

31. Heitz-Mayfield LJ, Huynh-Ba G. History of treated periodontitis and smoking as risks for implant therapy. Int J Oral Maxillofac Implants. 2009;24 Suppl:39-68. 